\title{
Acute Myeloid Leukemia (AML) Detection Using AlexNet Model
}

\author{
Maneela Shaheen, ${ }^{1}$ Rafiullah Khan $\mathbb{D},{ }^{1}$ R. R. Biswal $\left(\mathbb{D},{ }^{2}\right.$ Mohib Ullah, ${ }^{1}$ \\ Atif Khan $\mathbb{D}^{3},{ }^{3}$ M. Irfan Uddin, ${ }^{4}$ Mahdi Zareei $\mathbb{D}^{2},{ }^{2}$ and Abdul Waheed $\mathbb{D D}^{5,6}$ \\ ${ }^{1}$ Institute of Computer Science and Information Technology, The University of Agriculture, Peshawar, Pakistan \\ ${ }^{2}$ Tecnologico de Monterrey, School of Engineering and Sciences, Zapopan, Mexico \\ ${ }^{3}$ Department of Computer Science, Islamia College Peshawar, Peshawar, KP, Pakistan \\ ${ }^{4}$ Institute of Computing, Kohat University of Science and Technology, Kohat, Pakistan \\ ${ }^{5}$ Department of Information Technology, Hazara University Mansehra, Mansehra 21120, Pakistan \\ ${ }^{6}$ School of Electrical and Computer Engineering, Seoul National University, Seoul 08826, Republic of Korea
}

Correspondence should be addressed to Rafiullah Khan; rafiyz@gmail.com and Mahdi Zareei; m.zareei@ieee.org

Received 31 December 2020; Accepted 18 May 2021; Published 28 May 2021

Academic Editor: Dan Selisteanu

Copyright (c) 2021 Maneela Shaheen et al. This is an open access article distributed under the Creative Commons Attribution License, which permits unrestricted use, distribution, and reproduction in any medium, provided the original work is properly cited.

\begin{abstract}
Acute Myeloid Leukemia (AML) is a kind of fatal blood cancer with a high death rate caused by abnormal cells' rapid growth in the human body. The usual method to detect AML is the manual microscopic examination of the blood sample, which is tedious and time-consuming and requires a skilled medical operator for accurate detection. In this work, we proposed an AlexNet-based classification model to detect Acute Myeloid Leukemia (AML) in microscopic blood images and compared its performance with LeNet-5-based model in Precision, Recall, Accuracy, and Quadratic Loss. The experiments are conducted on a dataset of four thousand blood smear samples. The results show that AlexNet was able to identify $88.9 \%$ of images correctly with $87.4 \%$ precision and $98.58 \%$ accuracy, whereas LeNet-5 correctly identified $85.3 \%$ of images with $83.6 \%$ precision and $96.25 \%$ accuracy.
\end{abstract}

\section{Introduction}

Leukemia is a hematological disorder and type of cancer that weakens the human immune system by generating malignant White Blood Cells (WBC) [1, 2]. Leukemia is considered as one of the fatal cancers with a high death rate [3]. Leukemia is usually classified based on myelogenous or lymphoblastic disorders of the WBCs. If the affected cells are lymphoblastic, then the leukemia is called Acute Lymphoblastic Leukemia (ALL). If the affected WBCs are monocytes and granulocytes, then the leukemia will be called Acute Myeloid Leukemia (AML) [2].

Leukemia is a blood cancer resulting from an abundance of abnormal white blood cells in humans [2]. Usually, a hematology analyzer is used to diagnose leukemia through manual counting. Cell classification usually depends on the morphological characteristics of the cells and requires a skilled medical operator. This procedure can be time-consuming, tedious, and costly [3]. Moreover, the manual analyzer may sometimes lead to the incorrect counting and classification of leukocytes [4]. Undoubtedly, this manual examination mechanism can be replaced by machinelearning-based automated techniques that can save precious time and significantly reduce human effort and error $[5,6]$.

Deep learning algorithms are powerful and versatile algorithms used efficiently in significant research areas such as medical image processing, supercomputing, investment modeling, and fraud detections [7, 8]. Convolutional Neural Network $(\mathrm{CNN})$ is a popular subcategory of deep learning algorithms, specially designed for visual pattern recognition [9]. LeNet-5 is a CNN-based algorithm that uses seven different layers, including maximum pooling, convolutional, and Fully Connected (FC) [10]. Similarly, AlexNet is a prominent CNN-based algorithm [11] that uses eight 
different layers, including maximum pooling, convolutional, and FC [12]. AlexNet is declared the best neural network model for image classification in the Large Scale Visual Recognition Challenge (2012) [13].

In this regard, Thanh et al. [14], Snehal and Daware [15], Liang et al. [16], and Tiwari et al. [17] proposed CNN-based models for leukemia disease detection. Shafique and Tehsin [2], Mishra et al. [18, 19], and Tuba et al. [20] worked on Acute Lymphatic Leukemia (ALL) detection. In contrast, Su et al. [21] and Agaian et al. [22] worked on Acute Myeloid Leukemia (AML) detection. As a limited amount of work is done on Acute Myeloid Leukemia (AML) detection, this work addressed the problem of automatic detection of Acute Myeloid Leukemia (AML) using CNN.

AlexNet algorithm is a competent and well-known deep learning algorithm that can be used efficiently in significant research areas, especially medical image processing [11]. AlexNet can analyze and detect important features from different medical images such as CT scans, X-rays, MRI, PET, ultrasound, and hematological images.

In this work, we proposed an AlexNet-based classification model to detect Acute Myeloid Leukemia (AML) in microscopic blood images and compared its performance with LeNet-5-based model in Precision, Recall, Accuracy, and Quadratic Loss. The experiments are conducted on a dataset of four thousand blood smear samples. The results show that AlexNet was able to identify $88.9 \%$ of images correctly with $87.4 \%$ precision and $98.58 \%$ accuracy, whereas LeNet- 5 correctly identified $85.3 \%$ of images with $83.6 \%$ precision and $96.25 \%$ accuracy. In the future, we are planning to apply the AlexNet architecture for other types of leukemia cell detection, such as Acute Lymphatic Leukemia (ALL), to get high accuracy.

This paper is structured as follows. Section 2 discusses a literature review. Section 3 discusses the material and methods used in this work, such as the dataset description, AlexNet architecture, and performance evaluation techniques. Experimental results are discussed in Section 4, and conclusions and future work are discussed in Section 5.

\section{Background}

2.1. White Blood Cells (WBCs). WBCs (leukocytes) are the human immune system cells that defend the human body against disease, infections, and foreign bodies [23]. WBCs are produced in the bone marrow and derived from hematopoietic stem cells. There are five types of WBCs based on their functional and physical characteristics: lymphocytes, monocytes, eosinophils, basophils, and neutrophils. A brief introduction of all five types of WBCs is as follows:

Lymphocytes create antibodies against various microorganisms such as viruses, bacteria, and other potentially harmful invaders. There are further subtypes of lymphocytes, including B cells, T cells, and natural killer cells.

Monocytes have a longer lifespan, and they help to break down bacteria. The monocyte cell's primary purpose is to present a piece of germ to the $\mathrm{T}$ cell to identify it again in future attacks.

Eosinophils rise in the response of disease of nervous systems, collagen, spleen, parasitic infection, and different kinds of allergies, that is, hives and hay fever. However, their primary target is parasites such as tapeworms and hookworms.

Basophils are mainly responsible for antigen and allergic response by releasing histamine (an indication for allergy) that causes the blood vessels to dilate and help the human body for immune response.

Neutrophils act as the first line of defense in case of infection. Their primary purpose is to fight and kill the fungus or bacteria. They are a prevalent cell type seen in the early stages of inflammation, and their activity and death form Pus in the human body.

2.2. Types of Leukemia. Leukemia is a hematological disorder and type of blood cancer that weakens the human immune system by generating a surplus amount of malignant WBCs (White Blood Cells) $[1,2]$. Leukemia is usually classified into four major classes based on myelogenous or lymphoblastic disorders: Acute Myeloblastic (Myeloid) Leukemia (AML), Chronic Myeloblastic (Myeloid) Leukemia (CML), Acute Lymphoblastic (Lymphocytic) Leukemia (ALL), and Chronic Lymphoblastic (Lymphocytic) Leukemia (CLL). A brief introduction of all four major types of Leukemia is as follows [24]:

$A M L$ is the most common type of leukemia that usually occurs in both adults and children. It affects the WBCs of the human body and causes them to form abnormally. The abundance of abnormal WBCs in the human body causes acute cancers.

ALL is the most common type of cancer in childhood but can also occur in adults. ALL is caused due to the increased lymphocytes (a kind of WBCs) in the human body.

$C M L$ is a cancer of WBCs in which immature WBCs or blast cells are formed and multiply uncontrollably in a human body. CML is mostly affecting adults.

$C L L$ is a kind of cancer that affects B lymphocytes or $\mathrm{B}$ cells. $\mathrm{B}$ cells help the human body fight against infection; however, the cancerous B cells cannot fight infections. CLL usually affects older people.

\section{Related Work}

AL-Dulaimi et al. conducted a detailed survey WBCs classification techniques for identifying fatal diseases such as blood cancer, AIDS, and leukemia using microscopic images of hematology reports. They also pointed out some crucial challenges in the WBC classification process regarding WBCs structure, image quality, cell morphology, the classification's time complexity, and others [25]. Othman et al. worked on the classification of WBC using feedback and feedforward propagation neural networks. After analyzing 
blood cells achieved from telescopic images, the 16 most important elements of that cell were fed as input to the neural network. They trained the neural network with 50\% data after segmentation, while the rest of the data are used for testing. They achieved a $96 \%$ accuracy in classifying WBCs [26]. Lin et al. pointed out that recognizing the WBCs is difficult due to uneven color and irregular illuminations of blood smear images. They proposed a hybrid model composed of histogram distribution, K-means clustering, watershed algorithm, and CNN for WBCs classification with improved accuracy. They reported that their proposed hybrid model achieved 95.81\% accuracy [27]. Umamaheswari and Geetha briefly explained leukemia with all its subtypes and different image segmentation methods used in the machine learning domain to identify leukemia in microscopic images. They concluded their reviews with two significant findings: (i) Machine-learning- and imageprocessing-based hybrid techniques could offer better results in leukemia detection. (ii) A benchmark dataset is needed to find improvements in the methods proposed from time to time [28].

Thanh et al. proposed a CNN-based classification model for fast detection of acute leukemia disease. In the proposed method, the first classification process is covered in the experimental results, representing an outstanding achievement by differentiating abnormal and normal cells. Their proposed method achieved $96.6 \%$ accuracy in leukemia cell classification [14]. Daware proposed an in-depth CNN segmentation-based technique to identify leukemia blood cells in microscopic images. Their proposed methods could classify WBCs in blood smear images with $93.94 \%$ accuracy [15]. Liang et al. proposed a combined CNN- and RNNbased blood cell microscopic image classification model to solve the reliance link problem between significant features of images mark and images. They concluded that the proposed CNN and RNN combined model is more precise and accurate than other CNN-based models [16]. Tiwari et al. proposed Double Convolutional Layer Neural Network(DCLNN-) based blood cells detection model and compared its performance with SVM and Naïve Bayes-based models. They showed that their proposed DCLNN model is more accurate than SVM and Naïve Bayes models [17]. Negm et al. proposed a Decision Support System (DSS) to classify the leukemia cells in microscopic images using $\mathrm{K}$-means clustering and panel selection approach. They conducted experiments with various public and benchmark datasets, and after investigations, they verified their results with expert pathologists. They reported that their system achieved 99.517\% accuracy [29].

AlexNet algorithm is a competent and well-known deep learning algorithm that can be used efficiently in significant research areas, especially medical image processing [11]. AlexNet uses eight different layers, including maximum pooling, convolutional, and FC [12]. AlexNet can analyze and detect important features from different medical images such as CT scans, X-rays [30], MRI [31], PET [32], ultrasound [33], and hematological images [34].
3.1. Materials and Methods. This work aims to process and analyze the Acute Myeloid Leukemia smear images to provide an automatic technique to support the medical activity that enables pathologists to segment and recognize Acute Myeloid Leukemia (AML). The research methodology is based on blood smear image analysis for AML classification, using computer vision techniques. The WBCs have five subcategories called monocytes, lymphocytes, basophils, eosinophils, and neutrophils; however, for diagnostics of AML subclass of WBCs, myeloid is used. Therefore, to detect and classify AML, myeloid bodies are easily identifiable from blood smear images, as their nuclei do not look the same from the background and other blood cells. The myeloid, which is affected, is called a myeloid cell, which has additional morphological changes with the syndrome's increasing cruelty. Myeloid is present in regular shape and compact nucleus with having normal and continuous edges. Otherwise, the myeloid has shape irregularities and small nooks/holes in the cytoplasm. Therefore, the proposed procedure will extract the myeloid from the blood smear image, and then CNN features are extracted to classify it as normal or myeloid. We compared the performance of AlexNet with LeNet-5 [35] in terms of Precision, Recall, F-Measure, Accuracy, and Mean Square Error or Quadratic Loss.

The experiments are conducted on a workstation with a 3.1 GHz Core i5 CPU and 8 GB RAM in MATLAB 2018a environment with Deep Learning toolbox [36]. The blood smear pictures are collected from a reputable tertiary care hospital and publicly available microscopic peripheral blood images. In this section, we discuss the dataset, preprocessing steps, AlexNet model, and evaluation parameters.

3.1.1. Dataset. The dataset used in this study comprises four thousand blood smear acute myeloid samples gathered from two different sources, that is, a reputable tertiary care hospital of Peshawar, Pakistan, and publicly available microscopic peripheral blood images released by Acevedo et al. [37]. The dataset consists of 4000 images having 1500 normal monocytes, 1500 abnormal monocytes, and 1000 lymphocytes. The data are preclassified by the experts of the field. The properties of the dataset are available in Table 1.

3.1.2. Data Preprocessing. Data preprocessing is a critical step used to check the data for experiments; before image analysis, the images should be prepared for a good outcome. In our experiments, we resize every image into a $256 \times 256$ resolution image. Then they are converted to a $227 \times 227$ pixel image to feed it to the first layer of the AlexNet model.

3.1.3. AlexNet Architecture. The AlexNet model comprises five convolutional, three maximum pooling, and three FC layers. The AlexNet model needs a $227 \times 227$ resolution image as an input, and then ReLU (Rectified Linear Unit) activation function is applied to remove nonlinearity. After 
TABLe 1: Dataset properties.

\begin{tabular}{lc}
\hline Size of images & $960 \times 1080$ pixels \\
\hline Color model & RGB \\
Total classes & 3 \\
Normal monocytes & 1500 \\
Abnormal monocytes & 1500 \\
Lymphocytes & 1000 \\
\hline
\end{tabular}

that, the images are downsampled to extract rich features using pooling layers. The first FC layer is then used to flatten the feature vector, which drops out $50 \%$ of the features. The remaining features are then used for AML disease identification. Each Fully Connected layer contains 4096 neurons. The last FC layer is known as the output layer and will produce 1000 outputs. These 1000 outputs will be passed through the SoftMax activation function. SoftMax activation function or normalized exponential function is used to normalize the network's output to a probability distribution over predicted output classes. In the last phase, we trained and tested our CNN model, which classified different leukemia types that are either normal or abnormal. We apply 30 epochs on training with 5880 iterations. Figure 1 shows the AlexNet model structure, while Table 2 shows the parameters and names of all layers used in the AlexNet model.

3.1.4. Evaluation Parameters. The proposed model's performance is compared with the LeNet-5 model in Accuracy, Precision, Recall, and F-Measure. Precision represents the number of identified images belonging to the correct classes, and Recall represents how the total images are correctly recognized in their respective classes. F-Measure is the harmonic mean of Precision value and Recall value [38, 39]. We also assess the models' performance in Mean Square Error or Quadratic Loss [40].

$$
\begin{aligned}
\text { Precision } & =\frac{\mathrm{TP}}{\mathrm{TP}+\mathrm{FP}}, \\
\text { Recall } & =\frac{\mathrm{TP}}{\mathrm{TP}+\mathrm{FN}}, \\
F-\text { Measure } & =2 \times \frac{\text { Precision } \times \text { Recall }}{\text { Precision }+ \text { Recall }}, \\
\text { Accuracy } & =\frac{\mathrm{TP}+\mathrm{TN}}{\mathrm{TP}+\mathrm{FP}+\mathrm{TN}+\mathrm{FN}}, \\
\text { Quadratic Loss } & =\frac{\sum_{i=1}^{n}\left(x_{i}-x_{i}^{\prime}\right)^{2}}{n} .
\end{aligned}
$$

\section{Results and Discussion}

This research aims to improve the automatic detection of Acute Myeloid Leukemia (AML) in microscopic blood smear images. We proposed AlexNet-based CNN model for accurate detection of AML cells and compared its performance with the LeNet-5-based CNN model. LeNet-5 model is comprised of two convolutional, two maximum pooling, and three FC layers. In contrast, the AlexNet model has five convolutional, three maximum pooling, and three FC layers. The experiments are conducted with the preclassified dataset of 4000 images gathered from a reputable tertiary care hospital of Peshawar, Pakistan, and publicly available microscopic peripheral blood images. The data are preclassified by the experts of the field. The models' performance is evaluated in Precision, Recall, F-Measure, Accuracy, and Mean Square Error or Quadratic Loss.

Based on the experiments' results, the AlexNet-based model was able to correctly classify $88.9 \%$ of images with $87.4 \%$ precision, whereas LeNet-5 was able to classify $85.3 \%$ of images correctly with $83.6 \%$ Precision. Similarly, the F-Measure of LeNet-5-based model is recorded as 0.844 , whereas, in the case of AlexNet, the F-Measure value is 0.881 . The comparison of both LeNet-5- and AlexNet-based models in Precision, Recall, and F-Measure is illustrated in Table 3 and Figure 2.

Similarly, for the accuracy rate of both LeNet- 5 and AlexNet models, three classes are illustrated in Table 4 and plotted in Figure 3. According to the results, the accuracy of AlexNet in detecting normal monocytes was 98.88\%, whereas LeNet-5 gave $96.69 \%$ accuracy for the same class. Similarly, in detecting abnormal monocytes, the accuracies of AlexNet and LeNet-5 were $97.9 \%$ and $95.49 \%$, respectively, whereas, in the case of lymphocytes detection, the accuracies of both AlexNet and LeNet-5 models were 98.96\% and $96.78 \%$, respectively. The overall average accuracies of both AlexNet and LeNet-5 models for the whole dataset are $98.58 \%$ and $96.32 \%$.

In the case of AlexNet, the accuracy rate in model training starts from $30 \%$ and reaches $98.5 \%$ at 30 epochs, while LeNet-5 accuracy begins at 30\% and achieves $96.32 \%$. Similarly, both models' Quadratic Loss was nearly equal to zero at the 5800th iteration and 30 epochs. The performance compression of both models in Accuracy and Quadratic Loss is shown in Figures 4 and 5.

Based on the results, the overall performance of the AlexNet-based model is found to be satisfactory compared to LeNet-5-based model in terms of Precision, Recall, and Accuracy. Moreover, the Quadratic Loss of the AlexNetbased model decreased with each iteration and reached almost zero at the 5800th iteration. Based on the models' 

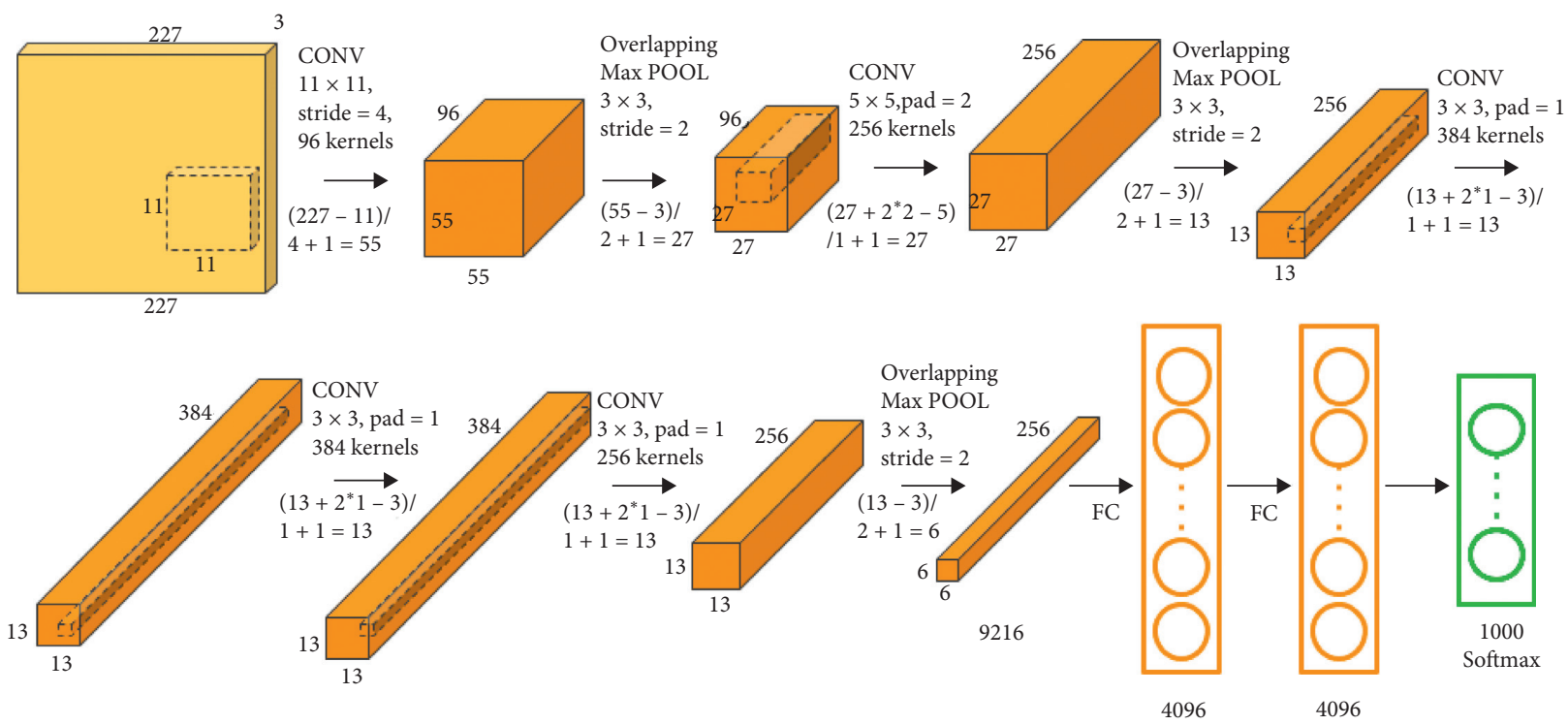

FIgURE 1: Structure of AlexNet model [12].

Table 2: Parameters detail of AlexNet model.

\begin{tabular}{lc}
\hline Algorithm & AlexNet \\
\hline Maximum number of epochs & 30 \\
Convolutional layers & ReLU activation function \\
Number of iterations & 5580 \\
Fully Connected layers & SoftMax activation function \\
Dataset & 4000 images \\
Training data & $70 \%$ \\
Testing data & $30 \%$ \\
\hline
\end{tabular}

Table 3: Performance comparison of both LeNet-5- and AlexNet-based models in Precision, Recall, and F-Measure.

\begin{tabular}{lccc}
\hline Model & Precision & Recall & F-Measure \\
\hline LeNet-5 & 0.836 & 0.853 & 0.844 \\
AlexNet & 0.874 & 0.889 & 0.881 \\
\hline
\end{tabular}

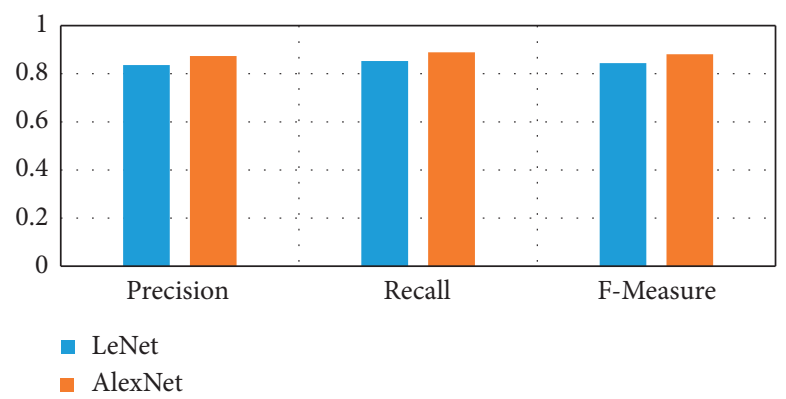

Figure 2: Performance comparison of both LeNet-5- and AlexNet-based models in Precision, Recall, and F-Measure.

TABle 4: Performance comparison of both LeNet-5- and AlexNet-based models in terms of Accuracy in each class.

\begin{tabular}{lcccc}
\hline & Normal monocytes & Abnormal monocytes & Lymphocytes & Average \\
\hline LeNet-5 & 0.9669 & 0.9549 & 0.9678 & 0.9632 \\
AlexNet & 0.9888 & 0.979 & 0.9896 & 0.9858 \\
\hline
\end{tabular}




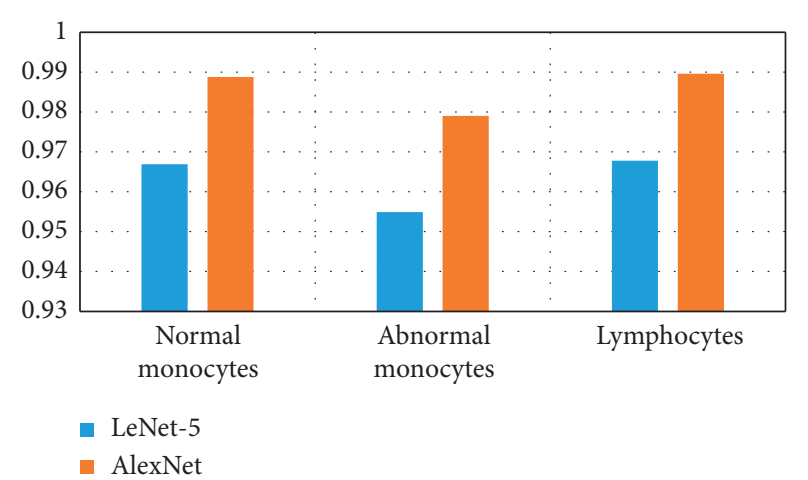

Figure 3: Performance comparison of both LeNet-5- and AlexNetbased models in terms of Accuracy in each class.

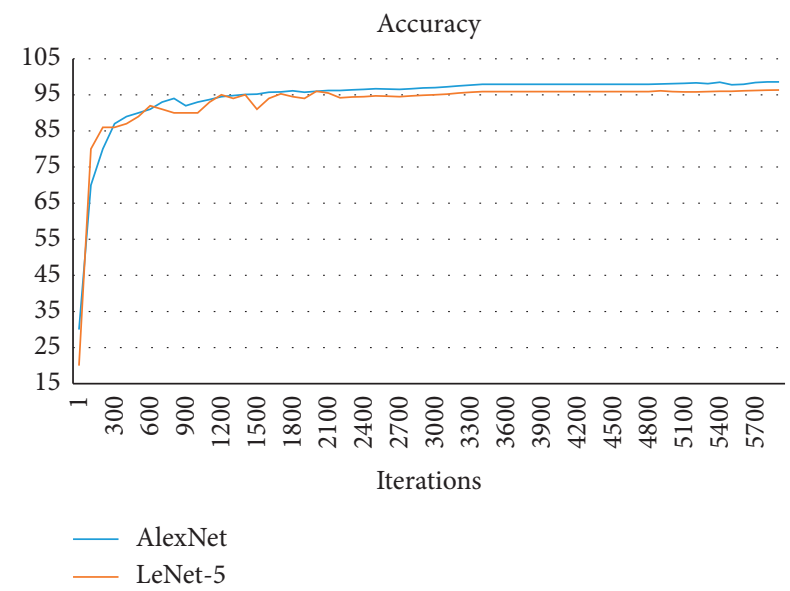

Figure 4: Performance comparison of both LeNet-5- and AlexNetbased models in Accuracy at every iteration.

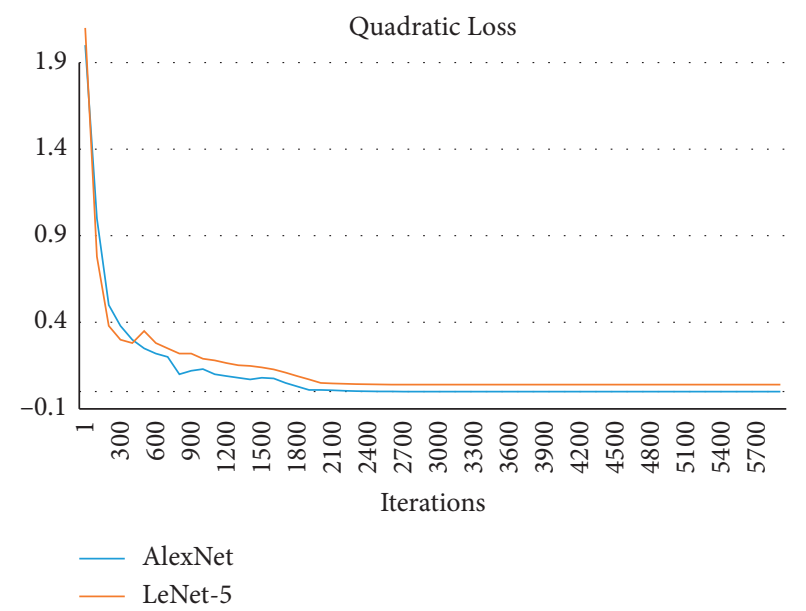

Figure 5: Performance comparison of both LeNet-5- and AlexNetbased models in Quadratic Loss at every iteration.

performance, it is safe to conclude that AlexNet-based models can be effectively used for hematological image analysis and AML detection.

\section{Conclusions and Future Work}

Acute Myeloid Leukemia (AML) is a hematological disorder and type of cancer that weakens the human immune system by generating malignant WBCs. In this work, we have proposed the AlexNet-based model to identify AML in microscopic blood images and compared its performance with the LeNet-5-based model in Precision, Recall, Accuracy, and Quadratic Loss. LeNet-5 model is composed of two convolutional, two maximum pooling, and three FC layers. In contrast, the AlexNet model has five convolutional, three maximum pooling, and three FC layers. Based on results, it is concluded that AlexNet performed well with high accuracy compared to LeNet-5-based model. AlexNet was able to classify $88.9 \%$ images correctly with $87.4 \%$ precision and 98.58\% accuracy, whereas LeNet-5 correctly identified $85.3 \%$ images with $83.6 \%$ precision and $96.25 \%$ accuracy.

AlexNet algorithm is a competent and well-known deep learning algorithm that can be used efficiently in significant research areas, especially medical image processing. AlexNet can analyze and detect important features from different medical images such as CT scans, X-rays, MRI, PET, ultrasound, and hematological images. In the future, we are planning to apply the AlexNet architecture for other types of leukemia cell detection, such as Acute Lymphatic Leukemia (ALL), to get high accuracy.

\section{Data Availability}

The data used to support the findings of this study are available at https://data.mendeley.com/datasets/snkd93bnjr/1.

\section{Conflicts of Interest}

The authors declare that there are no conflicts of interest regarding the publication of this paper.

\section{Acknowledgments}

This work was supported by Tecnologico de Monterrey, School of Engineering and Sciences, Mexico.

\section{References}

[1] R. B. Walter and E. H. Estey, "Selection of initial therapy for newly-diagnosed adult acute myeloid leukemia: limitations of predictive models," Blood Reviews, vol. 44, Article ID 100679, 2020.

[2] S. Shafique and S. Tehsin, "Acute lymphoblastic leukemia detection and classification of its subtypes using pretrained deep convolutional neural networks," Technology in Cancer Research \& Treatment, vol. 17, 2018.

[3] A. K. Dwivedi and A. Kumar, "Artificial neural network model for effective cancer classification using microarray gene expression data," Neural Computing and Applications, vol. 29, no. 12, pp. 1545-1554, 2018.

[4] S. S. Patel and O. K. Weinberg, "Diagnostic workup of acute leukemias of ambiguous lineage," American Journal of Hematology, vol. 95, no. 6, pp. 718-722, 2020. 
[5] N. George and L. Kurian, "An approach to detect acute myelogenous leukemia in blood microscopic images," IOSR Journal of Computer Engineering, vol. 99, pp. 1-4, 2016.

[6] S. Agaian, M. Madhukar, and A. T. Chronopoulos, "A new acute leukaemia-automated classification system," Computer Methods in Biomechanics and Biomedical Engineering: Imaging \& Visualization, vol. 6, no. 3, pp. 303-314, 2018.

[7] E. Nazari, A. H. Farzin, M. Aghemiri, A. Avan, M. Tara, and H. Tabesh, "Deep learning for acute myeloid leukemia diagnosis," Journal of Medicine and Life, vol. 13, pp. 382-387, 2020.

[8] P. Mamoshina, A. Vieira, E. Putin, and A. Zhavoronkov, "Applications of deep learning in biomedicine," Molecular Pharmaceutics, vol. 13, no. 5, pp. 1445-1454, 2016.

[9] P. Bharati and A. Pramanik, "Deep learning techniques-RCNN to mask R-CNN: a survey," in Proceedings of the Computational Intelligence in Pattern Recognition, pp. 657668, Springer, Xiamen, China, May 2020.

[10] C.-W. Zhang, M.-Y. Yang, H.-J. Zeng, and J.-P. Wen, "Pedestrian detection based on improved LeNet-5 convolutional neural network," Journal of Algorithms \& Computational Technology, vol. 13, 2019.

[11] M. Z. Alom, T. M. Taha, C. Yakopcic et al., "The history began from alexnet: a comprehensive survey on deep learning approaches," 2018, https://arxiv.org/abs/1803.01164.

[12] A. Krizhevsky, I. Sutskever, and G. E. Hinton, "Imagenet classification with deep convolutional neural networks," Communications of the ACM, vol. 60, no. 6, pp. 84-90, 2017.

[13] N. Kumar, N. Kaur, and D. Gupta, "Major convolutional neural networks in image classification: a survey," in Proceedings of International Conference on IoT Inclusive Life (ICIIL 2019), pp. 243-258, Chandigarh, India, June 2020.

[14] T. T. P. Thanh, C. Vununu, C. Vununu, S. Atoev, and S.-H. Lee, "Blood cell image classification using convolutional neural network," International Journal of Computer Theory and Engineering, vol. 10, no. 2, pp. 54-58, 2018.

[15] S. L. a. S. Daware, "Analysis of white blood cell segmentation techniques and classification using deep convolutional neural network for leukemia detection," HELIX, vol. 8, pp. 45194524, 2018.

[16] G. Liang, H. Hong, W. Xie, and L. Zheng, "Combining convolutional neural network with recursive neural network for blood cell image classification," IEEE Access, vol. 6, pp. 36188-36197, 2018.

[17] P. Tiwari, J. Qian, Q. Li et al., "Detection of subtype blood cells using deep learning," Cognitive Systems Research, vol. 52, pp. 1036-1044, 2018.

[18] S. Rodrigues, B. Majhi, and P. K. Sa, "Texture feature based classification on microscopic blood smear for acute lymphoblastic leukemia detection," Biomedical Signal Processing and Control, vol. 47, pp. 303-311, 2019.

[19] S. Mishra, B. Majhi, P. K. Sa, and L. Sharma, "Gray level cooccurrence matrix and random forest based acute lymphoblastic leukemia detection," Biomedical Signal Processing and Control, vol. 33, pp. 272-280, 2017.

[20] E. Tuba, I. Strumberger, N. Bacanin, D. Zivkovic, and M. Tuba, "Acute lymphoblastic leukemia cell detection in microscopic digital images based on shape and texture features," in Proceedings of the International Conference on Swarm Intelligence, pp. 142-151, 2019.

[21] J. Su, S. Liu, and J. Song, "A segmentation method based on HMRF for the aided diagnosis of acute myeloid leukemia," Computer Methods and Programs in Biomedicine, vol. 152, pp. 115-123, 2017.
[22] S. Agaian, M. Madhukar, and A. T. Chronopoulos, "Automated screening system for acute myelogenous leukemia detection in blood microscopic images," IEEE Systems Journal, vol. 8, no. 3, pp. 995-1004, 2014.

[23] H. Kutlu, E. Avci, and F. Özyurt, "White blood cells detection and classification based on regional convolutional neural networks," Medical Hypotheses, vol. 135, Article ID 109472, 2020.

[24] V. Lights, Leukemia, 2019, https://www.healthline.com/ health/leukemia.

[25] K. AL-Dulaimi, J. Banks, V. Chandran, I. Tomeo-Reyes, and K. Nguyen Thanh, "Classification of white blood cell types from microscope images: techniques and challenges," Microscopy Science: Last Approaches on Educational Programs and Applied Research, vol. 8, pp. 1-10, 2018.

[26] M. Z. Othman, T. S. Mohammed, and A. B. Ali, "Neural network classification of white blood cell using microscopic images," International Journal of Advanced Computer Science and Applications, vol. 8, pp. 99-104, 2017.

[27] L. Lin, W. Wang, and B. Chen, "Leukocyte recognition with convolutional neural network," Journal of Algorithms \& Computational Technology, vol. 13, 2018.

[28] D. Umamaheswari and S. Geetha, "Review on image segmentation techniques incorporated with machine learning in the scrutinization of leukemic microscopic stained blood smear images," in Proceedings of the International Conference on ISMAC in Computational Vision and Bio-Engineering, pp. 1773-1791, 2018.

[29] A. S. Negm, O. A. Hassan, and A. H. Kandil, "A decision support system for acute leukaemia classification based on digital microscopic images," Alexandria Engineering Journal, vol. 57, no. 4, pp. 2319-2332, 2018.

[30] S. Q. Salih, H. K. Abdulla, Z. S. Ahmed, N. M. S. Surameery, and R. D. Rashid, "Modified alexnet convolution neural network for covid-19 detection using chest x-ray images," Kurdistan Journal of Applied Research, vol. 10, pp. 119-130, 2020.

[31] S. Lu, S.-H. Wang, and Y.-D. Zhang, "Detection of abnormal brain in MRI via improved AlexNet and ELM optimized by chaotic bat algorithm," Neural Computing and Applications, vol. 99, pp. 1-13, 2020.

[32] T. Toyonaga, T. Shiga, K. Hirata et al., "Convolutional neural network (CNN) of MRI and FDG-PET images may predict hypoxia in glioblastoma," Journal of Nuclear Medicine, vol. 58, p. $699,2017$.

[33] J. Sun, T. Sun, Y. Yuan, X. Zhang, Y. Shi, and Y. Lin, “Automatic diagnosis of thyroid ultrasound image based on FCNAlexNet and transfer learning," in Proceedings of the 2018 IEEE 23rd International Conference on Digital Signal Processing (DSP), pp. 1-5, Shanghai, China, November 2018.

[34] H. A. Aliyu, M. A. A. Razak, R. Sudirman, and N. Ramli, "A deep learning AlexNet model for classification of red blood cells in sickle cell anemia," International Journal of Artificial Intelligence, vol. 9, pp. 221-228, 2020.

[35] M. Sharma, A. Bhave, and R. R. Janghel, "White blood cell classification using convolutional neural network," Advances in Intelligent Systems and Computing, in Soft Computing and Signal Processing, vol. 1, pp. 135-143, 2019.

[36] P. Kim, "Matlab deep learning, with machine learning," Neural Networks and Artificial Intelligence, vol. 130, p. 21, 2017.

[37] A. Acevedo, A. Merino, S. Alférez, Á. Molina, L. Boldú, and J. Rodellar, "A dataset of microscopic peripheral blood cell 
images for development of automatic recognition systems," Data in Brief, vol. 30, Article ID 105474, 2020.

[38] N. U. Haq, M. Ullah, R. Khan et al., "USAD: an intelligent system for slang and abusive text detection in PERSO-Arabicscripted Urdu," Complexity, vol. 2020, Article ID 6684995, 7 pages, 2020.

[39] R. Khan, A. Ahmad, A. O. Alsayed, M. Binsawad, M. A. Islam, and M. Ullah, "QuPiD attack: machine learning-based privacy quantification mechanism for PIR protocols in health-related web search," Scientific Programming, vol. 2020, Article ID 8868686, 11 pages, 2020.

[40] F. U. M. Ullah, A. Ullah, I. U. Haq, S. Rho, and S. W. Baik, "Short-term prediction of residential power energy consumption via CNN and multi-layer bi-directional LSTM networks," IEEE Access, vol. 8, pp. 123369-123380, 2019. 